\title{
Monitoring corrosion in oil pipelines using non-destructive test
}

\author{
Nsaif jasim Hadi ${ }^{1}$, Ahmed Atiyah Itwayya ${ }^{2}$, Ameer L. Saleh ${ }^{3}$, Hayder Tarar ${ }^{4}$ \\ ${ }_{1,4}^{1,4}$ Department of Petroleum Engineering, University of Misan, Iraq \\ ${ }^{2}$ General Company of South electricity distribution, Ministry of electricity, Iraq \\ ${ }^{3}$ Department of Electrical Engineering, University of Misan, Iraq \\ ${ }^{4}$ Directorate of Sewage Misan Province, Misan, Iraq
}

\begin{abstract}
The age of the pipelines is a factor in increasing the potential risk to corrosion in pipelines hence reduces the safety of pipelines, and gets pipelines more likely to explosion or breakage. Thus, that will threaten the safety of individuals and the environment. According to corrosion is the main reason that threatens the pipeline. The danger is not limited to residents, but it could be worrisome if any the leak happened near the water sources. Thus, it will be difficult to address quickly, and requires significant costs could reach billions of dollars. Therefore, it is important to study the reasons that could be caused the pipeline explosion to avoided any effects could be happened, for example, in this paper we will focused on corrosion that happened in oil pipe depending on ultrasonic test methods (UT) to test sample of pipeline. Ultrasonic test is a test which is done by transfer a high frequency pulse through test object and receives a reflected echoes by analyzing the reflected waves which will help us to determine the thickness and other material properties.
\end{abstract}

\section{Keywords: $\quad$ Corrosion, Wave, Reflection, Non-destructive test, ultrasonic test, C-scan}

\section{Corresponding Author:}

Ameer L. Saleh, Department of Electrical Engineering, University of Misan, Misan, Iraq.

Email: ameer-lateef@uomisan.edu.iq

\section{Introduction}

Many structures material (such as oil pipelines) when are located in hostile weather conditions. So, should be tested regularly for corrosion or cracks during their lifetime. Non-destructive test (NDT) is a material or objects testing without damaging it by using special equipment and methods. NDT is used in assessment of material in different areas such as aircraft, Space Shuttle, turbines and others. There are several types of NDT such as, ultrasonic, magnetic-particle, radiographic and eddy- current testing. This report will focus on the ultrasonic test methods (UT) to test sample of pipeline. Ultrasonic test is a test which is done by transfer a high frequency pulse through test object and receives a reflected echo, by analyzing the reflected wave; flaws, thickness and material properties can be determined [1,2].

This paper is divided into some sections, first of all, it will start with the introduction; secondly, in the Theory section some ultrasonic principle will be explained; thirdly, in the case study and data analysis section data which is supplied by a $\mu$ Oil will be analyzed and the finding will have sketched in form of C-Scan; Finally, the conclusion section will summarize some important points[3-5].

\section{Theory}

The damage in pipelines considered a directly threat to peoples, who living close to the places of extraction and transportation of oil and gas [6-8]. For example, a pipeline in the United States spread in more than 2.5 million miles beneath the soil, most of these lines are located in rural areas, and this is a serious risk that may affect the environment in the event of the leak or any other deterioration occurs. Furthermore, continuous development of local communities and urban areas now puts many people's homes closer to the pipelines 
from originally. That means the incident could increase as happened in 2010 during the San Bruno, California, Pipeline explosion [9] .

The danger is not limited to residents, but it could be worrisome if any the leak happened near the water sources [8]. Thus, it will be difficult to address quickly, and requires high costs [8]. Therefore, it is a very important to study the reasons that could be caused the pipeline explosion to avoided any effects could be happened, for example, in this part we will focused on corrosion. Therefore, we should deal with some of sound wave principles to be able using ultrasonic methods for testing defects in materials. Thus, there are basic rules we should study them before analyses or evaluate any ultrasonic signals such as.

\subsection{Wave propagation}

Typically, ultrasonic testing depends on vibration in materials which is also called "acoustic". All materials contain from atoms. They can be obliged to move with vibrating about their balance positions. When a material is exposed to an incident pressure then mechanical vibration will propagate through the material as a wave. For example, in solid materials, there are two modes of sound propagation [2].

$>$ Bulk waves which are divided into two types: longitudinal and shear waves. Figure 1 shows them.

$\checkmark$ Longitudinal waves: sometime called pressure or compression waves, in this mode the vibrations happen longitudinally i.e. parallel to wave direction as shown in figure (1).

$\checkmark$ Transverse or shear waves: in this mode molecules vibrate vertically to wave direction at a right angle as shown in figure (1).

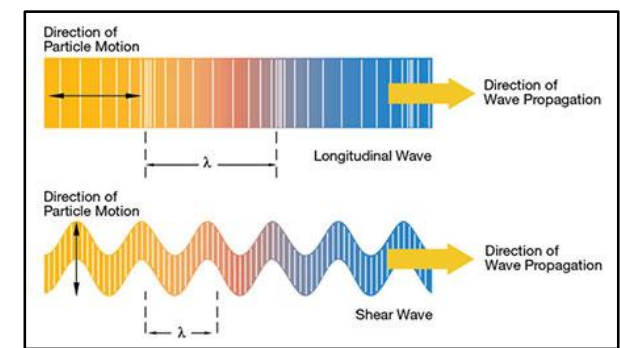

Figure 1. Longitudinal and shear waves propagation

Guided waves which are also divided into two types: plate and surface waves.

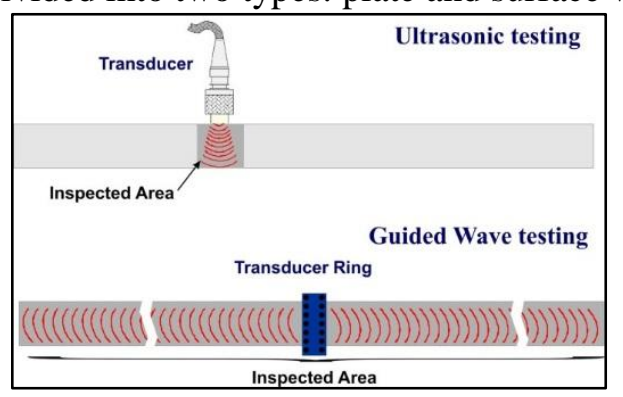

Figure 2. Guided waves propagation

Surface (or Rayleigh) waves: This mode is a compounding of longitudinal and shear waves result in elliptical motion as shown in figure (2) which travels on the surface of comparatively thick solid substance.

$\checkmark$ Plate / Lamb waves: In contrast to Surface wave plate waves can only produce in thick wavelength materials i.e. thin plated. They propagate parallel with the test surface across the material.

\subsection{Acoustic impedance}

One important parameter in ultrasound propagation is the ultrasonic impedance. It is a measurement of medium molecules resistance to a mechanical vibration. The acoustic impedance is proportion directly to the density of the medium and sound velocity in this material as shown in the equation (1) below [3] .

Where

$$
z=\rho * v
$$

- $\mathrm{p}=$ density

- $\mathrm{Z}=$ Acoustic impedance

- $\quad \mathrm{V}=$ velocity 
Acoustic impedance is important for:

$>$ Determination of transmission and reflection coefficient at the boundary of two materials having different acoustic impedances.

$>$ Design of ultrasonic transducers [2].

\subsection{Reflection and transmission coefficient}

When waves propagate between two materials part of these waves will reflected and the other will transmitted into the second material based on the material properties. Thus, "The ratio of ultrasonic wave intensity transmitted across an interface to the total wave energy incident on that interface" [2] which called transmission factor (coefficient) as illustrated in equation (2).

$$
(t)=\frac{2 Z_{2}}{Z_{2}+Z_{1}}
$$

"The ratio of reflected sound energy to incidence sound energy (intensity) at a reflecting surface, i.e. a surface separating two media of differing acoustic impedances, $Z_{1}$ and $Z_{2}[2]$ is called reflection coefficient which is represent in equation (3).

$$
(r)=\frac{Z_{2}-Z_{1}}{Z_{2}+Z_{1}}
$$

\subsection{Ultrasound attenuation}

It amounts of ultrasound wave energy which is lost at travelling across a medium. This loss occurs by the medium which absorbs the ultrasound energy and converted it to heat. Also there is other type of attention is scattering. According to [4] attenuation will increasing due to:

* Increasing the traveling distance.

* Increasing the impedance difference between the mediums.

* Increasing the transducer frequency.

\subsection{Refraction of ultrasound}

The deviation of ultrasound waves from its direction at the interface between two media where the ultrasound has different speed inside them is called refraction. The main cause of this phenomenon is changing the wavelength of the sound as it is travelling from the first media to the second with a fixed frequency. Figure (3) shows the wave refraction [3]. According to Snell's law as below.

Where:

$$
\frac{\sin \emptyset_{I}}{\sin \emptyset_{R}}=\frac{V_{c 1}}{V_{c 2}}
$$

$\mathrm{I}=$ angle of incidence

$\mathrm{R}=$ angle of refraction

$\mathrm{V} 1=$ sound velocity in medium 1

$\mathrm{V} 2=$ sound velocity in medium2

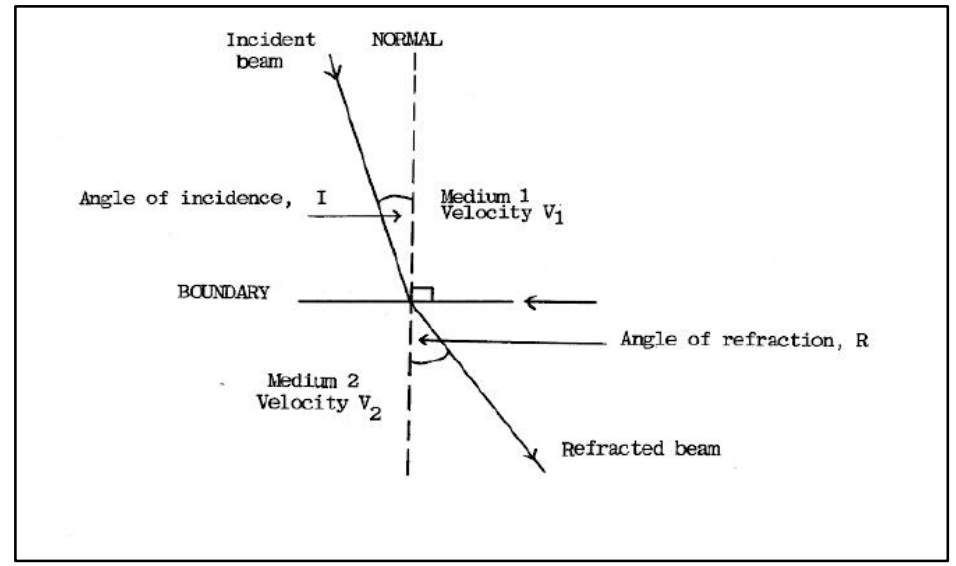

Figure 3. Wave refraction 


\subsection{Ultrasound test methods}

There are three different types of ultrasonic methods. Each one has specific features which will decide whether are suitable to test material by other[10,11].

\section{The pulse-echo method}

It is a method of ultrasonic test which is sent a perpendicular echo to the scanning surface and received a reflected wave from the back of the material by using one transducer figure (4). It is widely used to detect cracks and measure the thickness of materials. This method is useful for one side access objects [5].

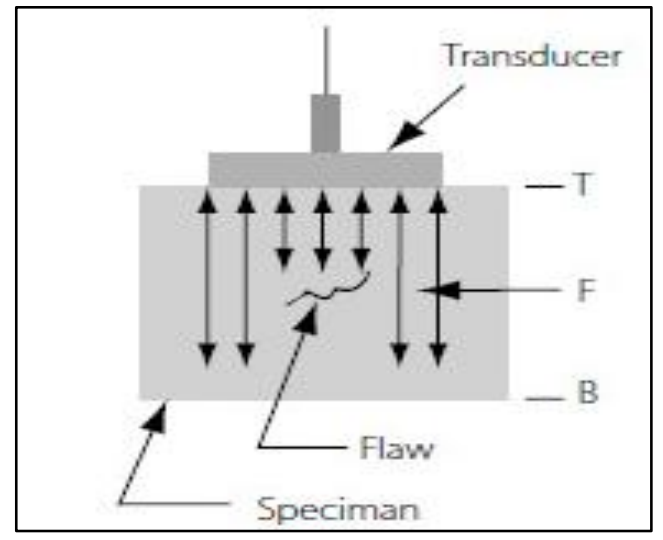

Figure 4. Pulse echo set up

\section{The pulse-through method}

Contrary to pulse-echo method this method is used two transducers in both sides of the material one of them send the echo and the other receive it. The two transducers should be at the same alignment which is sometime difficult to be achieved. It is used for velocity measurement and material characterization [5].

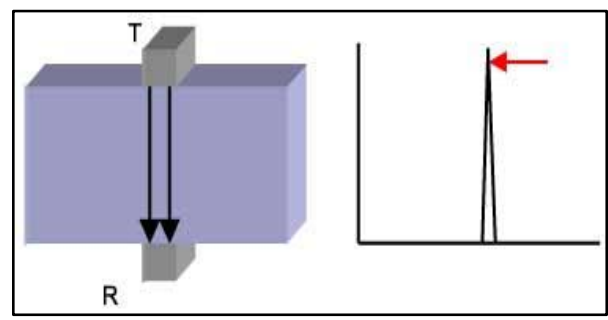

Figure 5. The pulse-through method

\section{The angle beams method}

In this method angled echo is sent through the material to improve the detectability of defects especially around the welded place figure (6). This method needs two transducers as the pulse-through method [2].

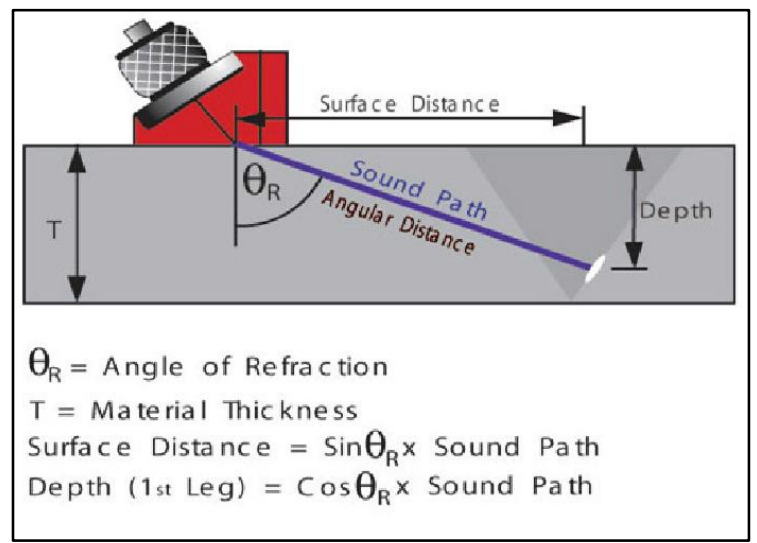

Figure 6. Angle beams method NDT 


\subsection{Data presentation}

According to [2] there are three types of data presentation. We could be present the information of ultrasonic test.

* A- SCAN: It is a presentation type which shows the ultrasonic energy on the vertical axis as a function of ultrasonic time travel within the material which is displayed on the horizontal axis. In AScan, by comparing an unknown reflected echo with a known one discontinuity can be discovered; the discontinuity size cold be estimated as well. The thickness of the material could be calculated by using the echo reflected time [5].

- B-SCAN: It is a cross sectional shows of the tested sample. Contrary to the A-Scan, the ultrasonic travel time is shown on the vertical axis, while the transducer linear position is shown on the horizontal axis. Using the B-scan, the thickness of the material and its estimated linear dimensions could be calculated [6].

- C-SCAN: The C-scan affords a plan type show of the size and location of test sample structures. The transducer scans pattern is parallel to the image plane. $\mathrm{C}$-scan is made using an automated data acquisition system. Generally, the collection of data is started with the A-scan and the signal amplitude recording at regular intervals while the transducer is being scanned over the test sample Figure (7) below shows C-Scan [7].

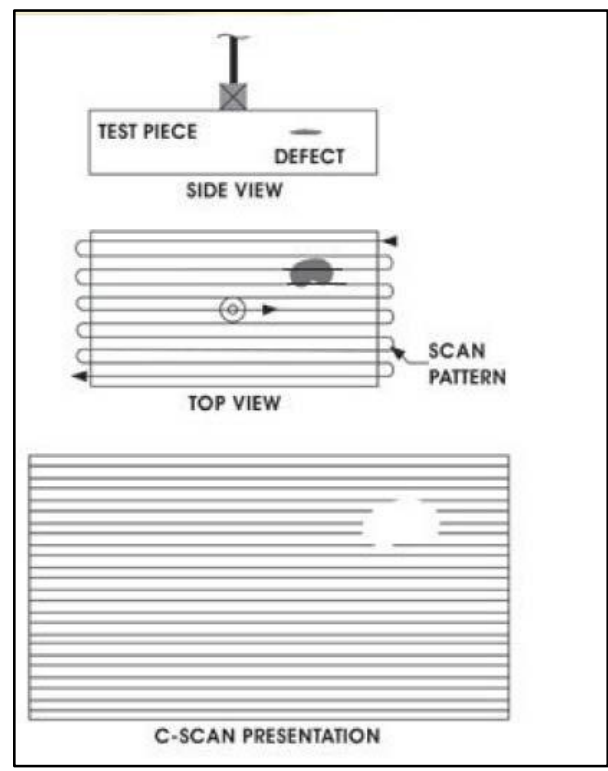

Figure 7. C-Scan presentations

\subsection{Ultrasonic general system component}

The ultrasonic system comprises of pulser / receiver, transducer, display and other component which is important to transmit and receive an ultrasound wave through an object as shown in figure (8) [2].

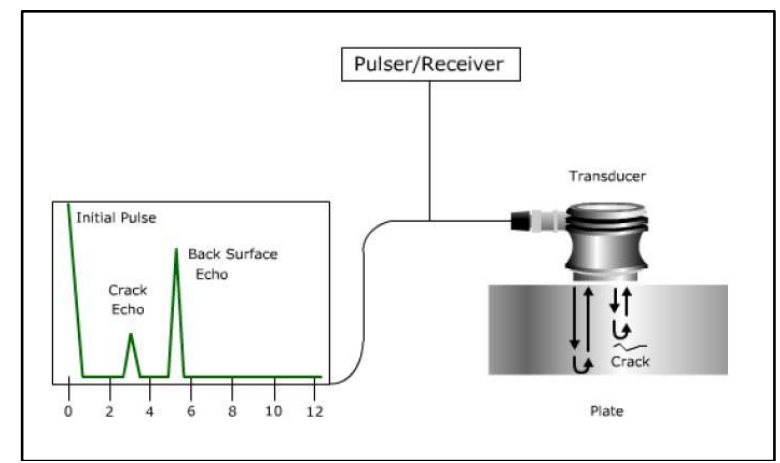

Figure 8. Ultrasonic system components [2] 


\section{Case study and data analysis (material and methods)}

\subsection{Analysis plan}

We have a steel sample of $5 \mathrm{~mm}$ thickness (ideal case) this sample divided into 49 parts as $(7 * 7)$ square grid as described in figure (9) and tested by ultrasound pulse echo measurements using a $10 \mathrm{MHz}$ centre frequency compression wave transducer. This test should be used to find below:

$\checkmark$ Internal cracks.

$\checkmark$ Internal and external corrosion.

$\checkmark$ Other defect such as pitting.

\begin{tabular}{|c|c|c|c|c|c|c|c|}
\hline & 1 & 2 & 3 & 4 & 5 & 6 & 7 \\
\hline A & A1 & A2 & A3 & A4 & A5 & A6 & A7 \\
\hline B & B1 & B2 & B3 & B4 & B5 & B6 & B7 \\
\hline C & C1 & C2 & C3 & C4 & C5 & C6 & C7 \\
\hline D & D1 & D2 & D3 & D4 & D5 & D6 & D7 \\
\hline E & E1 & E2 & E3 & E4 & E5 & E6 & E7 \\
\hline F & F1 & F2 & F3 & F4 & F5 & F6 & F7 \\
\hline G & G1 & G2 & G3 & G4 & G5 & G6 & G7 \\
\hline
\end{tabular}

Figure 9. Top view of a surface steel sample

\subsection{Pulse echo test}

We used standard pulse echo test with a single transducer (as illustrated figure 4). After recording the complete 49 A-scan, the data was analyzed and found 7 sorts of different shape of A-scan and these types divided into groups depend on the result of test.

* Ideal group: dominates the most of measurements points (Group one).

- Crack group: including air gap and pitting.

* Corrosion group: including back face and front face corrosion.

\subsection{Forming groups}

49 data of A-scans have been sorted into seven different groups (the similar data is arranged into one group) as shown below:

$>$ Group one $=$ A2, A3, B5, E1, E2, E3, F1, F2, F3, F4, G3, G4.

$>$ Group two $=$ A1, B1, C1, D1.

$>$ Group three $=$ A4, A5, A6, A7, B6, B7, G1, G2.

$>$ Group four = B2, B3, B4, C2, C5, D2, D5.

$>$ Group five $=\mathrm{C} 3, \mathrm{C} 4, \mathrm{D} 3, \mathrm{D} 4, \mathrm{E} 4, \mathrm{E} 5$.

$>$ Group six $=$ C6, C7, D6, D7, E6, E7, F5, F6, F7.

$>$ Group seven $=$ G5, G6, G7.

For each group an A-scan has been sketched using the Microsoft Excel program.

\subsection{Finding and analysis (results and discussion)}

\subsubsection{A-scan for group one}

We have a pulse echo result of A-scan for group one as shown in figure (10) below. It clearly shows the time $(\Delta \mathrm{T})$ between two echoes is equal, and by calculating the thickness for known velocity of Steel which is 6100 $\mathrm{m} / \mathrm{s}$ we can find it is equal to $5 \mathrm{~mm}$. that points out the signal travels along whole thickness of steel layer and back again without any errors in between. In addition, the signal has not any noise or attenuation or distortion in shape. Therefore, we can consider this group is ideal one.

$\Delta \mathrm{T}=$ Point 2 - Point 1

$\Delta \mathrm{T}=3.72 * 10^{-6}-2.08 * 10^{-6}$

$\Delta \mathrm{T}=1.64 * 10^{-6}$

$\mathrm{d}=\Delta \mathrm{T}^{*} \mathrm{C} / 2$

$\mathrm{d}=1.64 * 10^{-6} * 6100 / 2$ 


\section{$\mathrm{d} \approx 5.00 \mathrm{~mm}$}

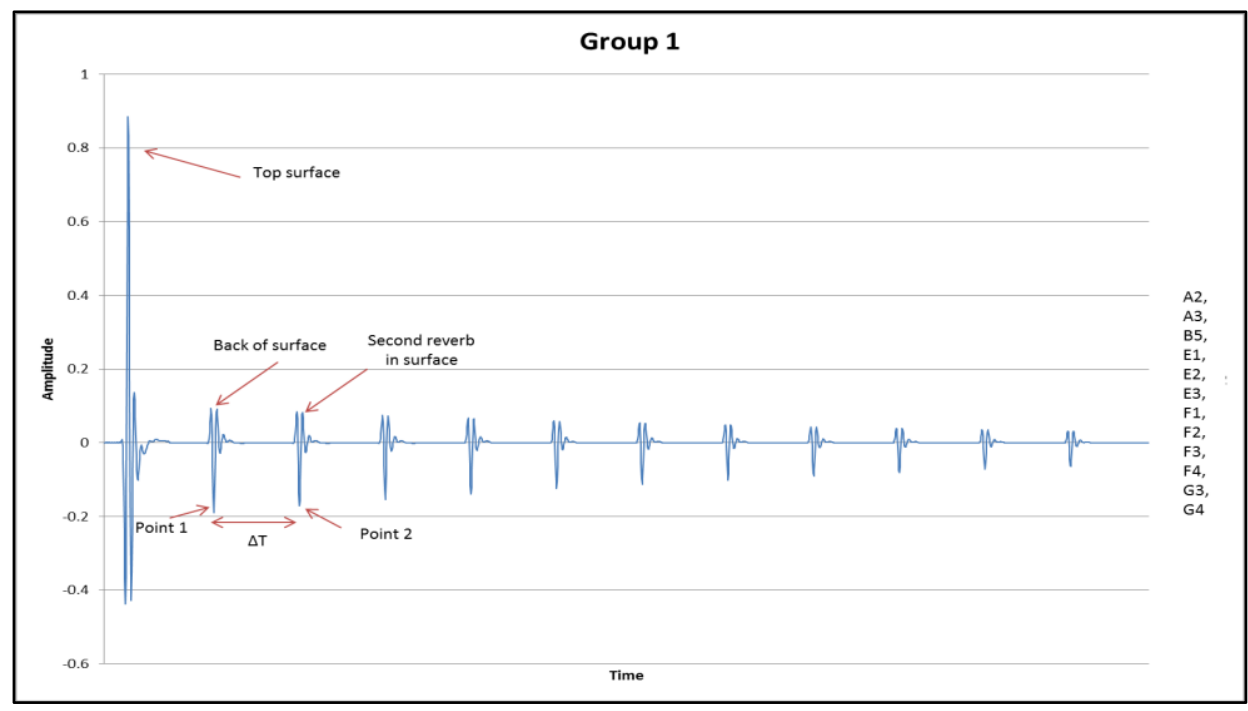

Figure 10. A-scan for group one

Observation:

Group one includes A2, A3, B5, E1, E2, E3, F1, F2, F3, F4, G3, and G4 samples are shown in C-Scan figure (11) below. According to setup graph which shows figure (12) it explains how echoes are generated from transducer and transmitted to steel layer, the first one is from the top surface, the second one is from the steel, and the third one is reverberated from steel.

\begin{tabular}{|c|c|c|c|c|c|c|}
\hline A1 & A2 & A3 & A4 & A5 & A6 & A7 \\
\hline B1 & B2 & B3 & B4 & B5 & B6 & B7 \\
\hline C1 & C2 & C3 & C4 & C5 & C6 & C7 \\
\hline D1 & D2 & D3 & D4 & D5 & D6 & D7 \\
\hline E1 & E2 & E3 & E4 & E5 & E6 & E7 \\
\hline F1 & F2 & F3 & F4 & F5 & F6 & F7 \\
\hline G1 & G2 & G3 & G4 & G5 & G6 & G7 \\
\hline
\end{tabular}

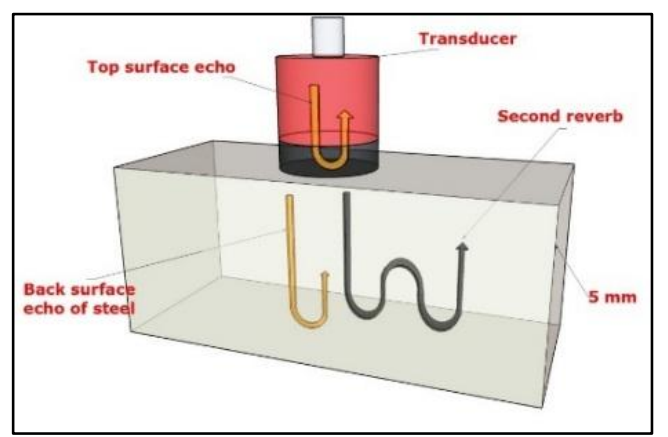

Figure 11. C-Scan of group one

Figure 12. Group one echoes

\section{The first echo}

Top surface echo is reflected back from the transducer shoe and top surface of steel layer. The amplitude is high because the large signal transmitted from the transducer to this layer and the reflection coefficient (r) at the boundary of steel-shoe is high due to the large difference between the acoustic impedances of the transducer shoe and steel material as explained below:

$Z=\rho^{*} c$ where

$\rho$ is the density of the material

$\mathrm{c}$ is the sound velocity in this material

$\mathrm{Z}_{\text {steel }}=\rho_{\text {steel }} * \mathrm{c}_{\text {steel }}$

When $\mathrm{f}=10 \mathrm{MHZ}$

$\mathrm{Z}_{\text {steel }}=7700 \mathrm{kgm}-3^{*} 6100 \mathrm{~ms}-1 \approx 47 \mathrm{M} \Omega$ that appeared very high if we comparing it with $\mathrm{Z}$ shoe which is small due to the properties of shoe's material.

Therefore, Reflection coefficient transducer $=\left(\mathrm{Z}_{\text {steel }}-\mathrm{Z}_{\text {shoe }}\right) /\left(\mathrm{Z}_{\text {steel }}+\mathrm{Z}_{\text {shoe }}\right)$ will be positive value. 


\section{The second echo}

Back surface echo is a reflected back from the back of steel layer. The amplitude reflection coefficient is $\left(Z_{2^{-}}\right.$ $\left.Z_{1}\right) /\left(Z_{2}+Z_{1}\right)$. Where $Z_{1}$ is the acoustic impedance of steel layer and $Z_{2}$ is the acoustic impedance of air. Due to $Z_{1}>Z_{2}$ the reflection coefficient will be a negative value. That will be caused all echoes are inverted and completely reflected $100 \%$ as well.

\subsubsection{A- Scan for Group Two}

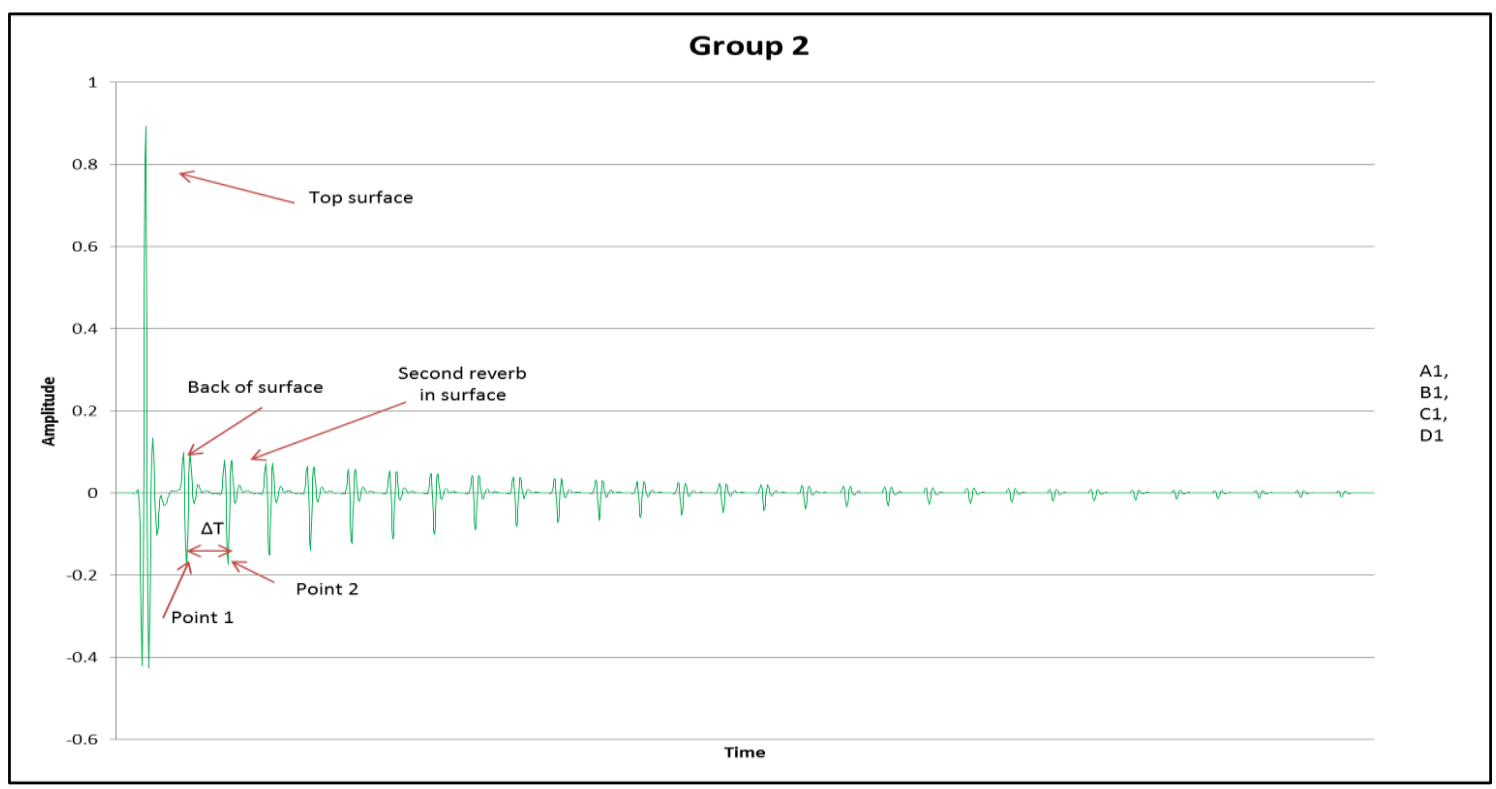

Figure 13. A-scan for group two

\section{Observation}

Group two includes (A1, B1, C1 and D1) samples are shown in C-Scan figure (14). When we look at the result of pulse echo of group two shown in figure (13) above, it clearly shows that $\Delta \mathrm{T}$ is similar at each time but it has a smaller value comparing with ideal one. Therefore, the thickness will be smaller too. As below:

$$
\begin{aligned}
& \Delta \mathrm{T}=\text { Point } 2 \text { - Point } 1 \\
& \Delta \mathrm{T}=1.76 * 10-6-1.11 * 10-6 \\
& \Delta \mathrm{T}=0.65 * 10-6 \\
& \mathrm{~d}=\Delta \mathrm{T} * \mathrm{C} / 2 \\
& \mathrm{~d}=0.65 * 10-6 * 6100 / 2 \\
& \mathrm{~d} \approx 2.00 \mathrm{~mm} \text { (crack location) }
\end{aligned}
$$

That is meaning there is a crack in this samples because the crack contains an air gap which is prevents the signal to be propagated to the end of steel layer and reflected back to the transducer. Therefore, we cannot know the rest of material after crack if has another flaws (e.g. back face corrosion). Based on Group two Ascan figure (15) it will illustrate how echoes are generated from the transducer and how it's transmitted to steel layer, the first echo is reflected from the top of steel surface to transducer, the second echo is reflected from the crack in the steel layer and the third one is reverberated from the crack and steel layer.

\begin{tabular}{|l|l|l|l|l|l|l|}
\hline A1 & A2 & A3 & A4 & A5 & A6 & A7 \\
\hline B1 & B2 & B3 & B4 & B5 & B6 & B7 \\
\hline C1 & C2 & C3 & C4 & C5 & C6 & C7 \\
\hline D1 & D2 & D3 & D4 & D5 & D6 & D7 \\
\hline E1 & E2 & E3 & E4 & E5 & E6 & E7 \\
\hline F1 & F2 & F3 & F4 & F5 & F6 & F7 \\
\hline G1 & G2 & G3 & G4 & G5 & G6 & G7 \\
\hline
\end{tabular}

Figure 14: C-scan of group two

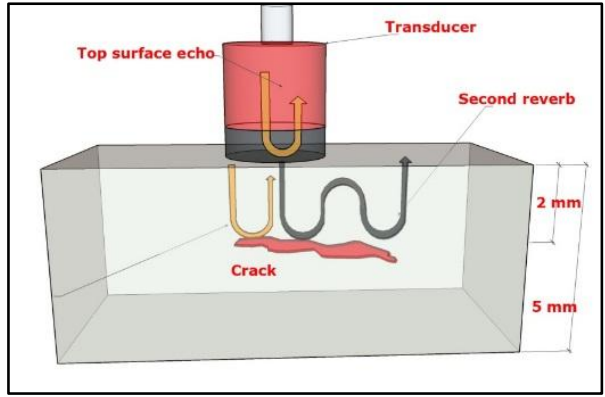

Figure 15. Group two echoes 


\subsubsection{A-scan for group three}

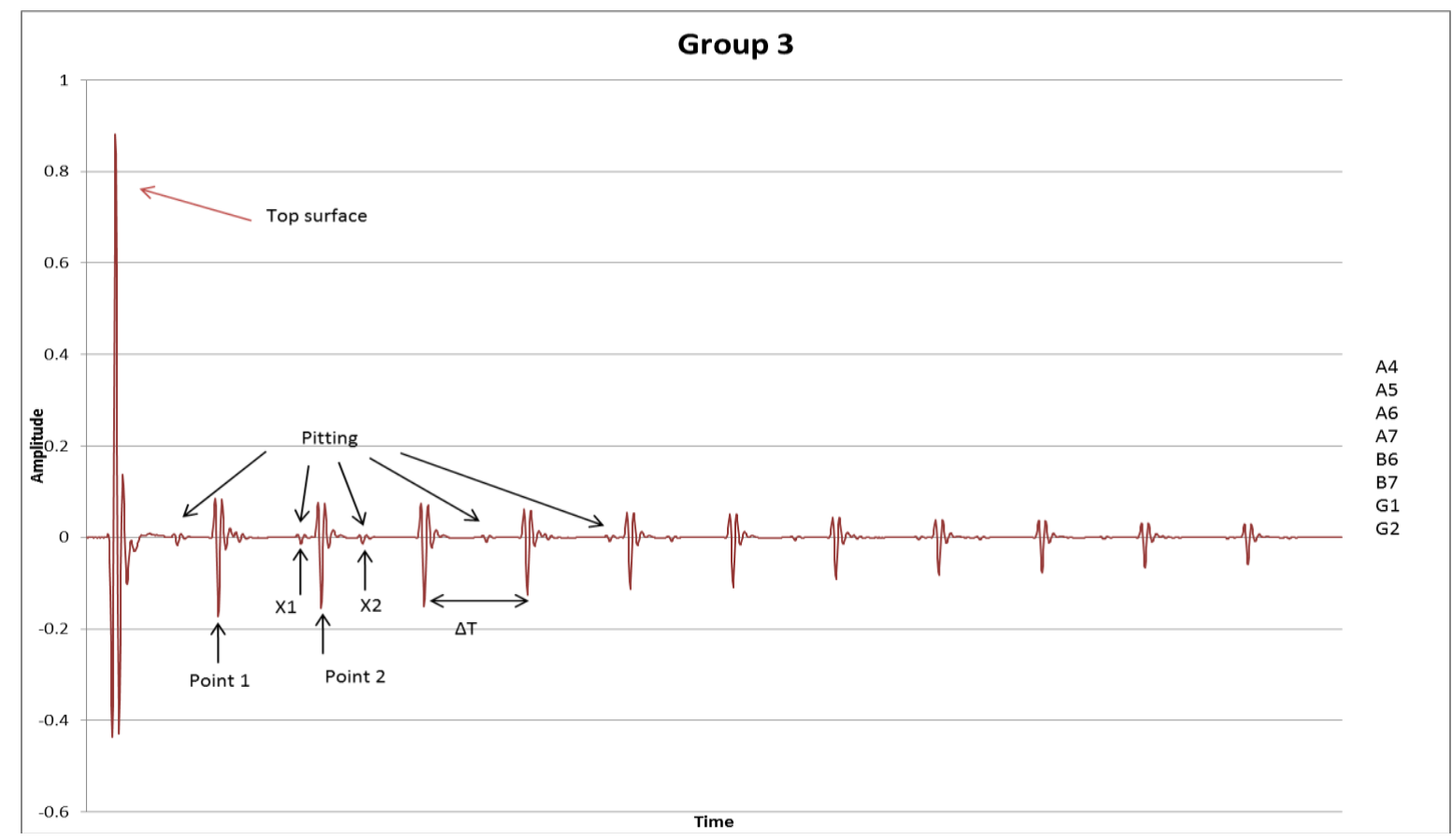

\section{Observation}

Figure 16. A-scan for group three

Group three includes A4, A5, A6, A7, B6, B7, G1 and G2 samples as shown in C-Scan figure (17) below. The echoes seem similar to the ideal case because it travelled from the transducer and return with same time which is taken by the echoes in ideal one as figure (16) above. We can notice there is a different in amplitude comparing with group one and these differences resulting from the existence of a small pitting located in this sample as shown in figure (18). This pitting is small in size, and in the same time the width of the sound beam is larger compared to the pit size, so the signal is continuing to propagate to back surface with losing some of its energy. There is only one pitting in the sample as it clear if we calculate the $\Delta \mathrm{T}$ between any two pitting echoes as elucidated in calculations below:

\begin{tabular}{|l|l|}
\hline \multicolumn{1}{|c|}{ Layer thickness } & \multicolumn{1}{c|}{ Location for pitting } \\
\hline$\Delta \mathrm{T}=$ Point $2-$ Point 1 & $\Delta \mathrm{T}=\mathrm{X} 1-$ Point 1 \\
$\Delta \mathrm{T}=3.72 * 10-6-2.08 * 10-6$ & $\Delta \mathrm{T}=3.38 * 10-6-2.08 * 10-6$ \\
$\Delta \mathrm{T}=1.64 * 10-6$ & $\Delta \mathrm{T}=1.29 * 10-6$ \\
$\mathrm{~d}=\Delta \mathrm{T} * \mathrm{C} / 2$ & $\mathrm{~d}=\Delta \mathrm{T} * \mathrm{C} / 2$ \\
$\mathrm{~d}=1.64 * 10-6 * 6100 / 2$ & $\mathrm{~d}=1.29 * 10-6 * 6100 / 2$ \\
$\mathrm{~d}=5.002 \mathrm{~mm}$ & $\mathrm{~d} \approx 3.93 \mathrm{~mm}$ \\
\hline
\end{tabular}

\begin{tabular}{|c|c|c|c|c|c|c|}
\hline A1 & A2 & A3 & A4 & A5 & A6 & A7 \\
\hline B1 & B2 & B3 & B4 & B5 & B6 & B7 \\
\hline C1 & C2 & C3 & C4 & C5 & C6 & C7 \\
\hline D1 & D2 & D3 & D4 & D5 & D6 & D7 \\
\hline E1 & E2 & E3 & E4 & E5 & E6 & E7 \\
\hline F1 & F2 & F3 & F4 & F5 & F6 & F7 \\
\hline G1 & G2 & G3 & G4 & G5 & G6 & G7 \\
\hline
\end{tabular}

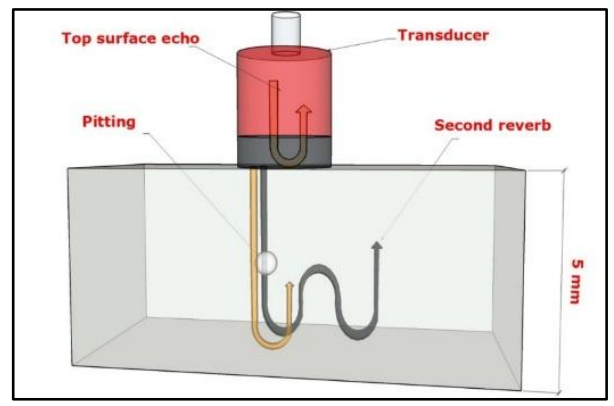

Figure 17. C-scan of group three

Figure 18. Group three echoes 


\subsubsection{A-scan for group (4)}

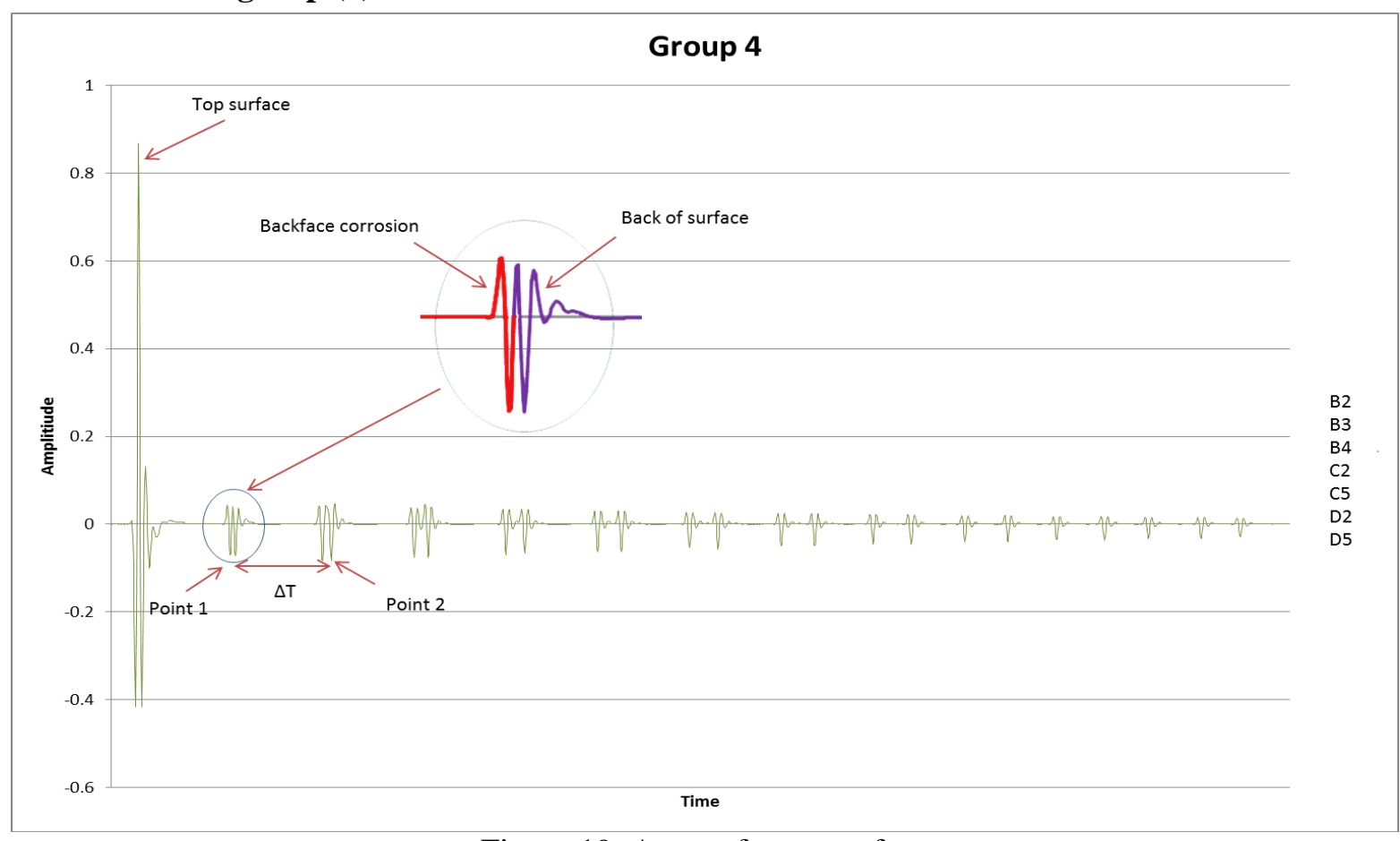

\section{Observation}

Figure 19. A-scan for group four

Group four includes B2, B3, B4, C2, C5, D2 and D5 samples as shown in C-Scan figure (20) and the set up shown in figure (21) below. By comparing it with the perfect one we can discover there are clearly difference in amplitude and shape of back surface echoes. This difference is the result of the back surface had corrosion in this group as shown in figure (19). These corrosions reflect the part of the signal which leads to attenuate the signal and thus reduce the amplitude of each echo. However, the distance between back surface echoes is the same and $\Delta \mathrm{T}$ is equal as in below simple calculations:

$\Delta \mathrm{T}=$ Point 2 - Point 1

$\Delta \mathrm{T}=3.56 * 10-6-2.00 * 10-6$

$\Delta \mathrm{T}=1.56 * 10-6$

$\mathrm{d}=\Delta \mathrm{T} * \mathrm{C} / 2$

$\mathrm{d}=1.56 * 10-6 * 6100 / 2$

$\mathrm{d} \approx 4.76 \mathrm{~mm}$ (back surface corrosion)

So

$5.00-4.76=0.25 \mathrm{~mm}$ (thickness of corrosion in the back face surface)

\begin{tabular}{|l|l|l|l|l|l|l|}
\hline A1 & A2 & A3 & A4 & A5 & A6 & A7 \\
\hline B1 & B2 & B3 & B4 & B5 & B6 & B7 \\
\hline C1 & C2 & C3 & C4 & C5 & C6 & C7 \\
\hline D1 & D2 & D3 & D4 & D5 & D6 & D7 \\
\hline E1 & E2 & E3 & E4 & E5 & E6 & E7 \\
\hline F1 & F2 & F3 & F4 & F5 & F6 & F7 \\
\hline G1 & G2 & G3 & G4 & G5 & G6 & G7 \\
\hline
\end{tabular}

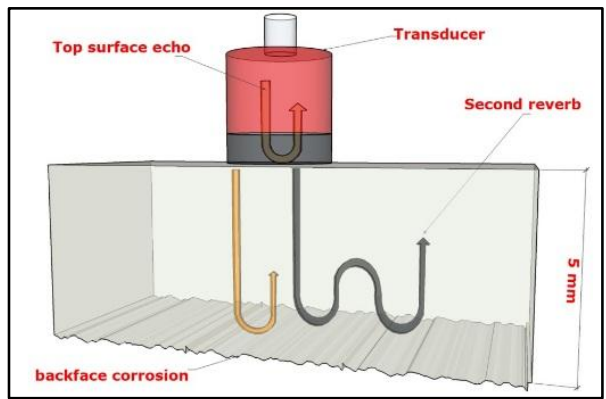

Figure 20. C-scan of group four

Figure 21. Group four echoes 


\subsubsection{A-scan for group five}

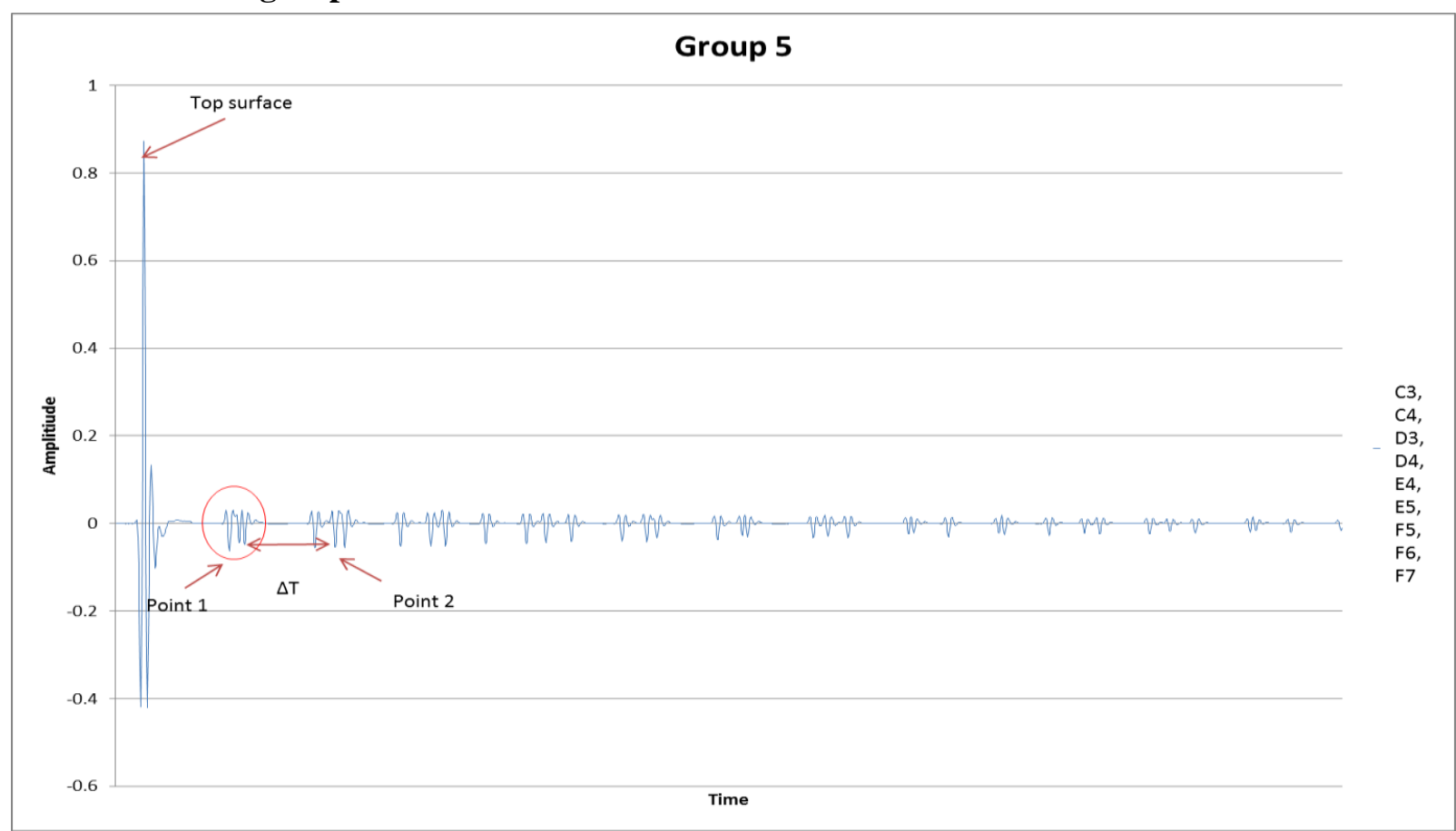

Figure 22. A-scan for group five

\section{Observation}

Group five includes C3, C4, D3, D4, E4 and E5 samples as shown in C-Scan figure (23) below. By comparing it with the ideal one we can notice there is a difference in amplitude and shape of echoes (especially, Back surface echo and second reverberation) as shown in figure (22) above. This variation is the result of the back surface had a bigger corrosion than group four as shown in figure (19). This corrosion reflects and scatters the large amount of the incidents signals which leads to reduce the amplitude of each echo as shown in figure (23), so the signal is attenuated. Alternatively, the distance between back surface echoes is the same and $\Delta \mathrm{T}$ is equal as in below simple calculations:

$\Delta \mathrm{T}=$ Point 2 - Point 1

$\Delta \mathrm{T}=3.22 * 10-6-1.83 * 10-6$

$\Delta \mathrm{T}=1.39 * 10-6$

$\mathrm{d}=\Delta \mathrm{T}^{*} \mathrm{C} / 2$

$\mathrm{d}=1.39 * 10-6 * 6100 / 2$

$\mathrm{d} \approx 4.24 \mathrm{~mm}$ (back surface corrosion)

Then

$5.00-4.24=0.75 \mathrm{~mm}$ (thickness of corrosion in the back face surface)

\begin{tabular}{|l|l|l|l|l|l|l|}
\hline A1 & A2 & A3 & A4 & A5 & A6 & A7 \\
\hline B1 & B2 & B3 & B4 & B5 & B6 & B7 \\
\hline C1 & C2 & C3 & C4 & C5 & C6 & C7 \\
\hline D1 & D2 & D3 & D4 & D5 & D6 & D7 \\
\hline E1 & E2 & E3 & E4 & E5 & E6 & E7 \\
\hline F1 & F2 & F3 & F4 & F5 & F6 & F7 \\
\hline G1 & G2 & G3 & G4 & G5 & G6 & G7 \\
\hline
\end{tabular}

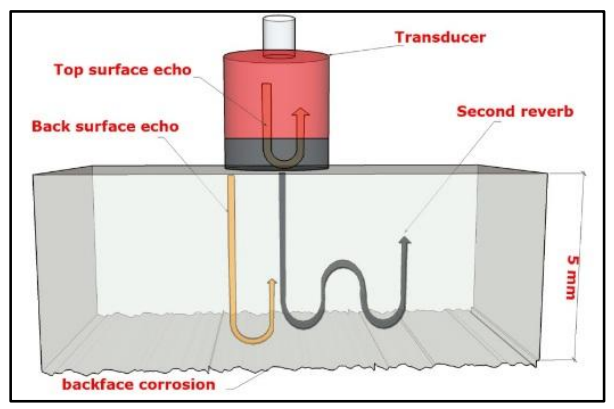

Figure 23. C-scan of group five

Figure 24. Group five echoes 


\subsubsection{A- scan for group six}

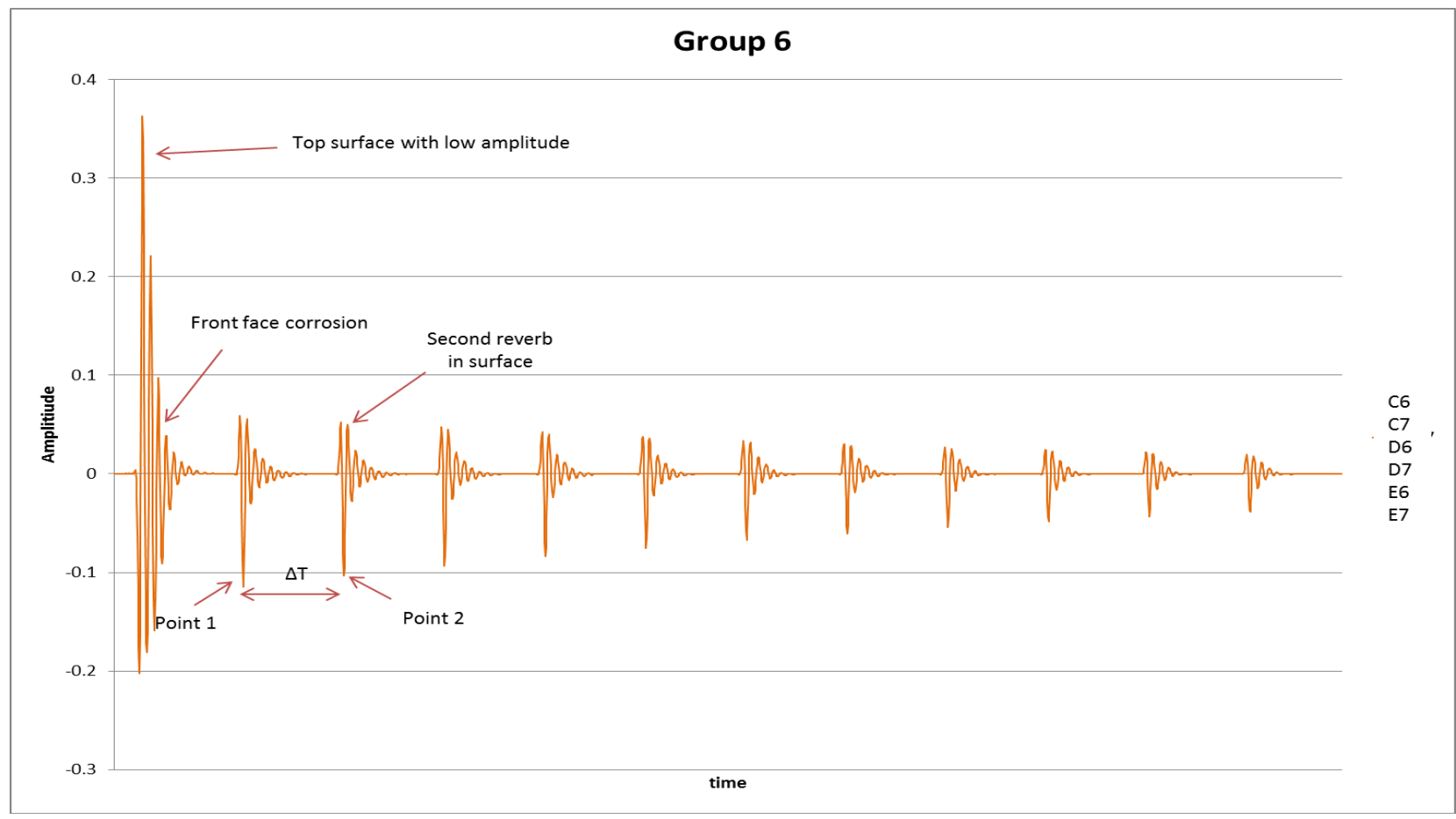

Figure 25: A-scan for group Six

\section{Observation}

Group six includes C6, C7, D6, D7, E6, E7, F5, F6 and F7 samples as shown in C-Scan figure (26) below. First of all, it is clearly there is changing in an amplitude of first echo (top surface) due to the surface of steel layer in this sample had a corrosion which led to scatter the signals into the different directions and prevents the reflection to the transducer directly as shown in figure (25) and the setup in figure (27). Despite of there is corrosion in the top surface of steel layer the thickness is not highly affected which indicates the corrosion depth is small. Below calculations will explain more:

$\Delta \mathrm{T}=$ Point 2 - Point 1

$\Delta \mathrm{T}=3.72 * 10^{-6}-2.08 * 10^{-6}$

$\Delta \mathrm{T}=1.64 * 10^{-6}$

$\mathrm{d}=\Delta \mathrm{T}^{*} \mathrm{C} / 2$

$\mathrm{d}=1.64 * 10^{-6} * 6100 / 2$

$\mathrm{d} \approx 5.00 \mathrm{~mm}$

\begin{tabular}{|l|l|l|l|l|l|l|}
\hline A1 & A2 & A3 & A4 & A5 & A6 & A7 \\
\hline B1 & B2 & B3 & B4 & B5 & B6 & B7 \\
\hline C1 & C2 & C3 & C4 & C5 & C6 & C7 \\
\hline D1 & D2 & D3 & D4 & D5 & D6 & D7 \\
\hline E1 & E2 & E3 & E4 & E5 & E6 & E7 \\
\hline F1 & F2 & F3 & F4 & F5 & F6 & F7 \\
\hline G1 & G2 & G3 & G4 & G5 & G6 & G7 \\
\hline
\end{tabular}

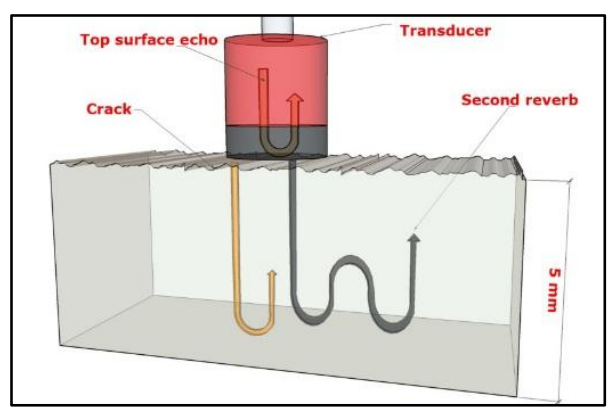

Figure 26. C-scan of group six

Figure 27. Group six echoes 


\subsubsection{A-scan for group Seven}

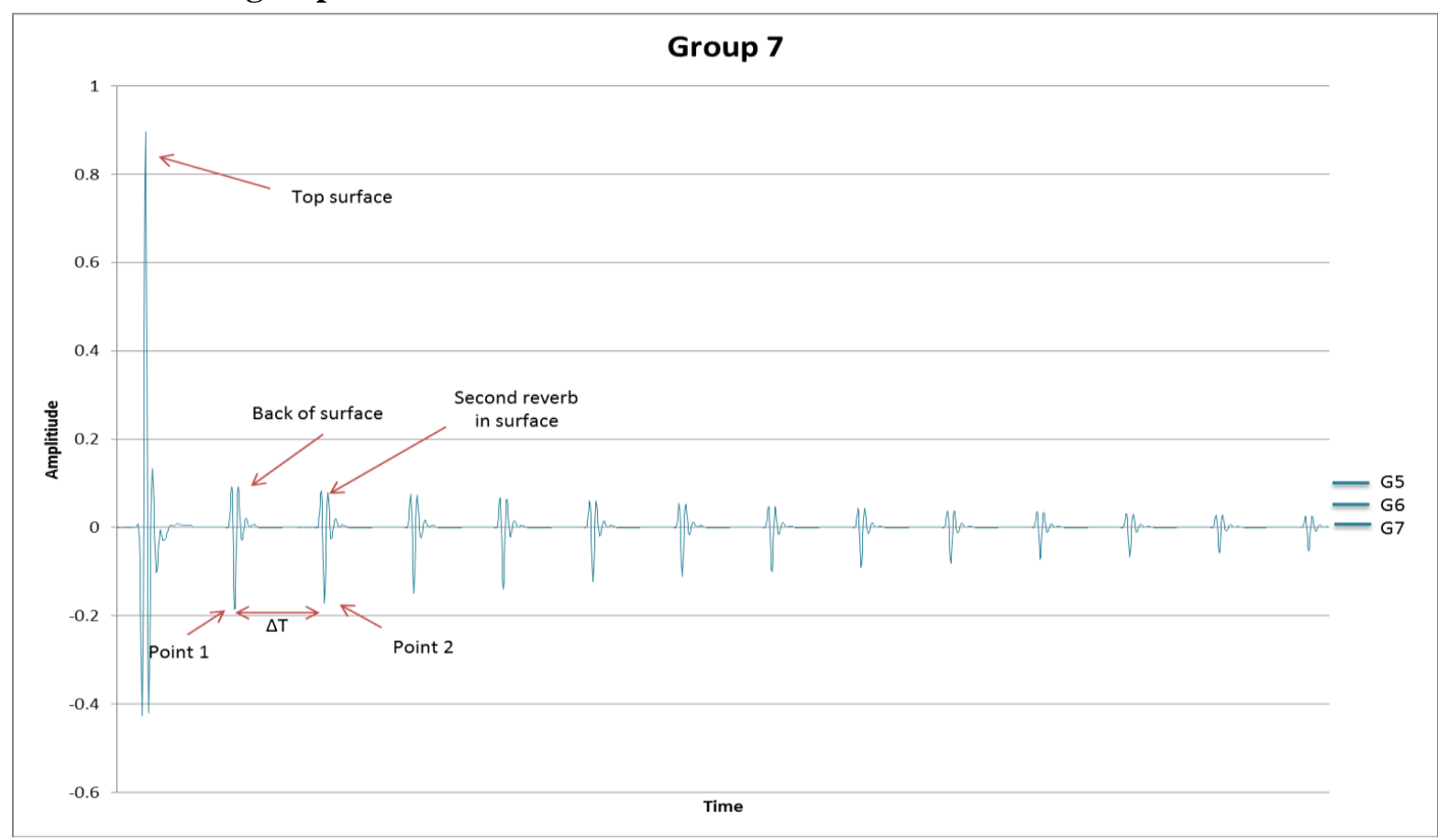

\section{Observation}

Figure 28. A-scan for group seven

Group seven includes G5, G6 and G7 samples as shown in C-Scan figure (29) below. When we look at the result of pulse echo of group seven shown in figure (28) above, it clearly shows that $\Delta \mathrm{T}$ is similar at each time but it has a smaller value comparing with ideal one. Therefore, the thickness will be smaller too. As below:

$$
\begin{aligned}
& \Delta \mathrm{T}=\text { Point } 2-\text { Point } 1 \\
& \Delta \mathrm{T}=3.40^{*} 10^{-6}-1.92 * 10^{-6} \\
& \Delta \mathrm{T}=1.48 * 10^{-6} \\
& \mathrm{~d}=\Delta \mathrm{T} * \mathrm{C} / 2 \\
& \mathrm{~d}=1.48 * 10^{-6} * 6100 / 2 \\
& \mathrm{~d} \approx 4.50 \mathrm{~mm} \text { (crack location) }
\end{aligned}
$$

That is means there is a crack in this sample and it prevents the signal to be propagated to end of layer and reflected to the transducer. Therefore, we cannot know the rest of material after crack if has another flaws (e.g. back face corrosion). Location of this crack explains in figure (30).

\begin{tabular}{|c|c|c|c|c|c|c|}
\hline a1 & a2 & a3 & a4 & a5 & a6 & a7 \\
\hline b1 & b2 & b3 & b4 & b5 & b6 & b7 \\
\hline c1 & c2 & c3 & c4 & c5 & c6 & c7 \\
\hline d1 & d2 & d3 & d4 & d5 & d6 & d7 \\
\hline e1 & e2 & e3 & e4 & e5 & e6 & e7 \\
\hline f1 & f2 & f3 & f4 & f5 & f6 & f7 \\
\hline g1 & g2 & g3 & g4 & G5 & G6 & G7 \\
\hline
\end{tabular}

Figure 29. C-scan of group seven

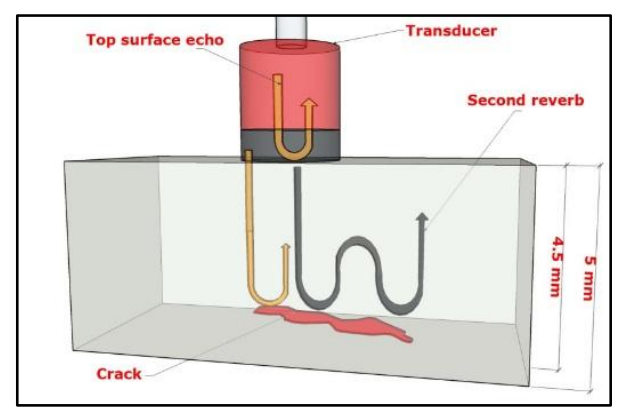

Figure 30. Group seven echoes 


\subsubsection{C-scan presentation to all groups}

As a result, for the findings above of all A-scan data analyses, these will be formed together in a C-scan graph to indicate more information about the test object as shown in figures $(31,32$ and 33) below.

\begin{tabular}{|c|c|c|c|c|c|c|}
\hline A1 & A2 & A3 & A4 & A5 & A6 & A7 \\
\hline B1 & B2 & B3 & B4 & B5 & B6 & B7 \\
\hline C1 & C2 & C3 & C4 & C5 & C6 & C7 \\
\hline D1 & D2 & D3 & D4 & D5 & D6 & D7 \\
\hline E1 & E2 & E3 & E4 & E5 & E6 & E7 \\
\hline F1 & F2 & F3 & F4 & F5 & F6 & F7 \\
\hline G1 & G2 & G3 & G4 & G5 & G6 & G7 \\
\hline
\end{tabular}

\begin{tabular}{|c|c|}
\hline \multicolumn{2}{|c|}{ Legend } \\
\hline & Ideal Group \\
\hline
\end{tabular}

Figure 31. C-Scan for ideal group

\begin{tabular}{|l|l|l|l|l|l|l|}
\hline A1 & A2 & A3 & A4 & A5 & A6 & A7 \\
\hline B1 & B2 & B3 & B4 & B5 & B6 & B7 \\
\hline & & & & & & \\
\hline C1 & C2 & C3 & C4 & C5 & C6 & C7 \\
\hline D1 & D2 & D3 & D4 & D5 & D6 & D7 \\
\hline E1 & E2 & E3 & E4 & E5 & E6 & E7 \\
\hline F1 & F2 & F3 & F4 & F5 & F6 & F7 \\
\hline G1 & G2 & G3 & G4 & G5 & G6 & G7 \\
\hline
\end{tabular}

\begin{tabular}{|l|l|}
\hline \multicolumn{2}{|c|}{ Legend } \\
\hline & Pitting \\
\hline & Crack at $2 \mathrm{~mm}$ \\
\hline & Crack at $4.5 \mathrm{~mm}$ \\
\hline
\end{tabular}

Figure 32. C-scan for internal crack groups

\begin{tabular}{|c|c|c|c|c|c|c|}
\hline A1 & A2 & A3 & A4 & A5 & A6 & A7 \\
\hline B1 & B2 & B3 & B4 & B5 & B6 & B7 \\
\hline C1 & C2 & C3 & C4 & C5 & C6 & C7 \\
\hline D1 & D2 & D3 & D4 & D5 & D6 & D7 \\
\hline E1 & E2 & E3 & E4 & E5 & E6 & E7 \\
\hline F1 & F2 & F3 & F4 & F5 & F6 & F7 \\
\hline G1 & G2 & G3 & G4 & G5 & G6 & G7 \\
\hline
\end{tabular}

\begin{tabular}{|c|c|}
\hline \multicolumn{2}{|c|}{ Legend } \\
\hline & Back face Corrosion $-0.25 \mathrm{~mm}$ \\
\hline & Back face Corrosion $-0.75 \mathrm{~mm}$ \\
\hline & Front face Corrosion \\
\hline
\end{tabular}

Figure 33. C-scan for internal and external corrosion groups

\section{Conclusion}

In conclusion, finding the defect at early stage could prevent a leak in oil pipeline. There are different NDT methods could be used to inspection materials; the most common one is the ultrasound test, which has different methods to do the test such as: Phased Array Ultrasonic Testing, Laser Ultrasonic Testing and others. In addition, there are many researches that are working to develop the use of Ultrasonic Testing technology. This paper has performed a complete data analysis of A-scan measurements for the steel pipeline sample of $5 \mathrm{~mm}$ thickness. Afterward, all findings in terms of corrosion, crack and pitting are gathered in multiple Cscans map to obtain a comprehensive form about the internal structure of the object. 


\section{References}

[1] F. Jiang, S. Liu, and H. Zhang, "Characterisation of cylindrical hidden corrosion using 2D analytical modelling of the surface magnetic field," Insight - Non-Destructive Testing and Condition Monitoring, vol. 60, no. 9, pp. 501-507, Sep. 2018.

[2] E. S. Ameh, S. C. Ikpeseni, and L. S. Lawal, "A Review of Field Corrosion Control and Monitoring Techniques of the Upstream Oil and Gas Pipelines," Nigerian Journal of Technological Development, vol. 14, no. 2, p. 67, Jan. 2018.

[3] R. Suwalak and C. Phongcharoenpanich, "Non-destructive determination of coconut oil using microwave dielectric constant sensing," Acta Horticulturae, no. 1256, pp. 59-62, Oct. 2019.

[4] N. Sciences, A. H. Dakheel, A. H. Dakheel, H. H. Abbas, and A. Info, "Intrusion detection system in gas-pipeline industry using machine learning,” Period. Eng. Nat. Sci., vol. 7, no. 3, pp. 1030-1040, 2019.

[5] S. Rashid, A. Ahmed, I. Al Barazanchi, and Z. A. Jaaz, "Clustering algorithms subjected to K-mean and gaussian mixture model on multidimensional data set," Period. Eng. Nat. Sci., vol. 7, no. 2, pp. 448-457, 2019.

[6] V. V. Thendral Tharmalingam, "An Efficient Convolutional Neural Network Based Classifier to Predict Tamil Writer,” Period. Eng. Nat. Sci., vol. 6, no. 1, pp. 285-295, 2018.

[7] S. Imashuku and K. Wagatsuma, "Non-destructive evaluation of alumina scale on heat-resistant steels using cathodoluminescence and X-ray-excited optical luminescence," Corrosion Science, vol. 154, pp. 226-230, Jul. 2019.

[8] S. Nešić, A. Kahyarian, and Y. S. Choi, "Implementation of a Comprehensive Mechanistic Prediction Model of Mild Steel Corrosion in Multiphase Oil and Gas Pipelines," CORROSION, vol. 75, no. 3, pp. 274-291, Dec. 2018.

[9] E. S. Ameh, S. C. Ikpeseni, and L. S. Lawal, "A Review of Field Corrosion Control and Monitoring Techniques of the Upstream Oil and Gas Pipelines," Nigerian Journal of Technological Development, vol. 14, no. 2, p. 67, Jan. 2018.

[10] Y. Lugovtsova and J. Prager, "Structural health monitoring of composite pressure vessels using guided ultrasonic waves," Insight - Non-Destructive Testing and Condition Monitoring, vol. 60, no. 3, pp. 139-144, Mar. 2018.

[11] H. A. M. Gamal, T. M. Zewail, and E.-S. Z. El-Ashtoukhy, "Effect of crude oil on the rate of diffusion-controlled corrosion in pipelines under turbulent flow conditions," Corrosion Reviews, vol. 36, no. 5, pp. 483-493, Sep. 2018. 